\title{
URINARY BLADDER CALCULUS WITH B/L MULTIPLE URETERIC CALCULI- A RARE COMPLICATION OF PRIMARY EXSTROPHY-EPISPADIAS COMPLEX CLOSURE
}

\author{
Anshul Garg1, Kumar Gaurav Mishra², Pawan Kumar³, Urvashi Mishra ${ }^{4}$
}

${ }_{1}^{1}$ MCH $3^{\text {rd }}$ Year Trainee, Department of Urology, IGIMS, Patna.

${ }^{2}$ MCH $1^{\text {st }}$ Year Trainee, Department of Urology, IGIMS, Patna.

${ }^{3}$ Senior Resident, Department of Urology, IGIMS, Patna.

${ }^{4} \mathrm{MS}$ (Obstetrics and Gynaecology), IGIMS, Patna.

HOW TO CITE THIS ARTICLE: Garg A, Mishra KG, Kumar P, et al. Urinary bladder calculus with B/L multiple ureteric calculi- A rare complication of primary exstrophy-epispadias complex closure. J. Evolution Med. Dent. Sci. 2017;6(56):4215-4216, DOI: $10.14260 /$ Jemds/2017/913

\section{PRESENTATION OF CASE}

We report a case of a 7-year-old male who had already undergone primary repair of exstrophy in neonatal period involving primary closure of the bladder and anterior abdominal wall. Since then he has had recurrent urinary symptoms, including suprapubic pain. Urine culture has never been conclusive, and he was managed with antibiotics both for prophylaxis and treatment. He presented to his GP with sharp suprapubic pain, severe enough to necessitate absence from school on several days.

On examination, he was found to have infraumbilical scar mark with epispadias and chordae.

His routine blood investigations, renal profile, serum electrolytes, serum calcium, serum parathyroid hormone levels were normal. His urine was normal on routine and microscopic examination and no growth was found on culture.

Treatment was started with testosterone to induce virilisation with encouraging results. IVU shows $\mathrm{B} / \mathrm{L}$ grade- 3 hydroureteronephrosis, urinary bladder almost completely occupied by radio dense large UB calculus with bilateral multiple ureteric calculi. Patient was managed by operative intervention in the form of open cystolithotomy.

Biochemical analysis of stone was positive for carbonate, calcium, oxalate. Patient was advised diet as per dietician's opinion. The patient is on regular followup from past 6 months and is doing well.

\section{DIFFERENTIAL DIAGNOSES}

1. Urinary Bladder Stone.

2. Ureteric Stone.

3. Lymph node calcifications.

\section{CLINICAL DIAGNOSIS}

Followup case of stage 1 exstrophy closure with B/L multiple ureteric calculi with giant bladder stone with epispadias.

\section{PATHOLOGICAL DISCUSSION}

Large bladder stone occupying whole of the bladder with two large right ureteric stones and four left ureteric stones were seen. Stone composition was $90 \%$ calcium oxalate monohydrate and $10 \%$ calcium carbonate.

Financial or Other, Competing Interest: None.

Submission 05-06-2017, Peer Review 02-07-2017,

Acceptance 08-07-2017, Published 13-07-2017.

Corresponding Author:

Kumar Gaurav Mishra,

Room No. 31, Mew MDH,

IGIMS Campus, Patna-14.

E-mail: gauravmishra.pmch@gmail.com

DOI: $10.14260 /$ jemds $/ 2017 / 913$
Stone disease in paediatric patients has genetic, metabolic, dietary and anatomic causes. There are numerous genetic causes of hypercalciuric nephrolithiasis alone that contribute to paediatric stone disease. It has been reported that $50 \%$ of those children with reconstructed bladders develop bladder stones in their lifetime. Urinary stasis, bacterial colonisation or infection with urea-splitting organisms, retained mucus and foreign bodies all can contribute to formation of bladder stones, most of which are struvite.

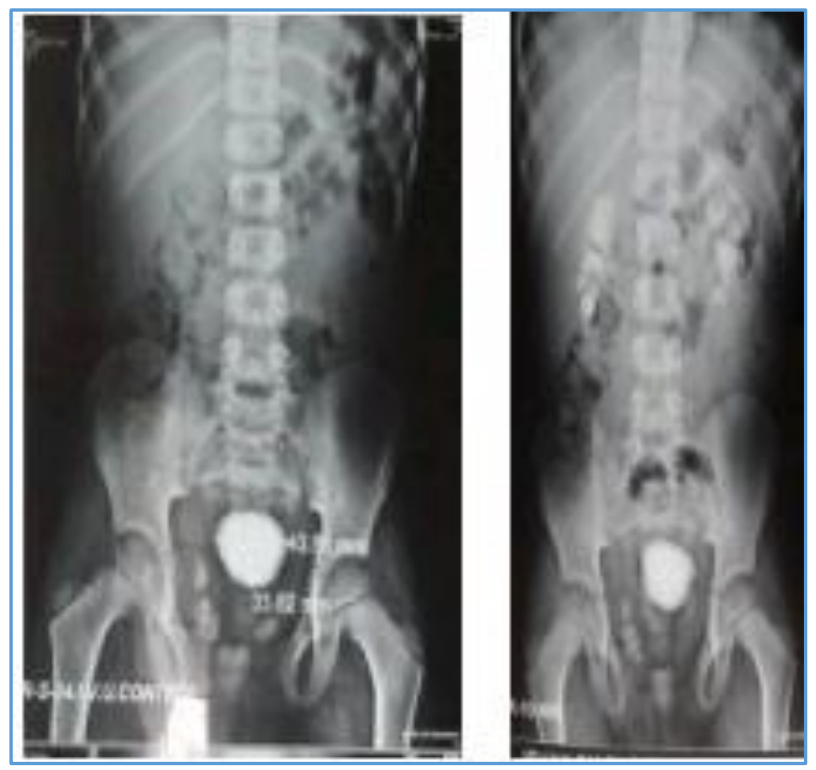

Figure 1. IVU, Giant bladder calculus with bilateral multiple ureteral calculi

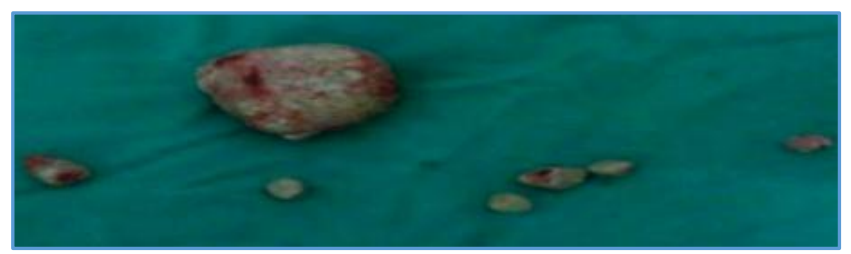

Figure 2. Giant vesical calculus with multiple ureteric calculi

\section{DISCUSSION}

The exstrophy of bladder is usually repaired in stages. Vesical calculus in EB repaired patients is known to occur. Many of the stones develop on the suture material used for the repair which is mostly non-absorbable. Bladder neck obstruction with resultant stasis and infection in EB repaired patients predisposes to stone formation. In a study of 530 patients with EB, $15 \%$ developed stones; white male population was the 
most commonly affected group and most calculi formed in the urinary bladder whether native or augmented by enterocystoplasty. It was also noted that the risk of stone formation in EB patients was associated with operative procedures like augmentation cystoplasty and bladder neck procedure to increase the outlet resistance; other risk factors being urinary infection, foreign bodies, vesicoureteral reflux and urinary stasis.

The condition is thought to be caused by incomplete development of the infra-umbilical part of the anterior abdominal wall, associated with incomplete development of the anterior wall of the bladder owing to delayed rupture of the cloacal membrane. Persistence of the cloacal membrane prevents medial mesenchymal ingrowth, causing the abdominal wall to remain lateral and the posterior bladder wall to be exposed to the external surface. Trigone of the bladder and ureteral openings are exposed and sometimes there is mild prolapse. The anterior abdominal wall defect involves the entire urethra and bladder neck. The pubic symphysis is always widened with diastasis of rectus abdominis. Umbilicus is low set. Frequently, there is omphalocoele which is confluent with exstrophic bladder.

Diagnostic evaluations of Exstrophy calculi include imaging, such as X-rays, ultrasound, CT scan. Plain X-rays of the abdomen, pelvis, and entire course of the bladder can be used to identify and measure radio-opaque stones. Direct visualisation modalities such as cystourethroscopy used for confirmation. Ultrasonography is a non-invasive diagnostic tool that may be useful for the screening of radio-opaque posterior UB and B/L ureteric calculi or to demonstrate an echogenic area with acoustic shadowing representing the calculi.

\section{Treatment and Management}

A 7-year-old male who had already undergone primary repair of EB in neonatal period at another hospital. Patient was incontinent and had history of dysuria, haematuria and intermittent episodes of fever with chills and rigors since one year. On examination, lower mid line abdominal scar mark was present with epispadias and chordae. The haemoglobin was $10.6 \mathrm{~g} / \mathrm{dL}$; TLC was $8,700 / \mathrm{mm}^{3}$, and s. creatinine $1.1 \mathrm{mg} / \mathrm{dL}$. Serum calcium, phosphate and uric acid levels were normal. IVU shows B/L grade-3 hydroureteronephrosis, urinary bladder almost completely occupied by radio-dense large UB calculus with bilateral multiple ureteric calculi. Open cyst lithotomy and bilateral ureterolithotomy with dilatation of bladder neck done under GA. Intraoperative findings showed normal capacity urinary bladder densely adhered to parities with stenosed bladder neck. Epispadias closure and bladder neck reconstruction was done at later date. Major complications of exstrophy closure are bladder prolapse, osteotomy non-union, leg length inequality and femoral nerve palsy. Minor complications include bladder outlet obstruction, urethrocutaneous fistula \& bladder stone. Stone formation after primary bladder closure is very rare.

Closure of bladder exstrophy is a safe surgery with an acceptable risk of complications. A critical review of outcomes provides insight to further refine the technique and manage complications when they develop.

Bladder exstrophy is a rare congenital anomaly(1) with 6:1 male-to-female ratio.(2,3) Urinary calculi formation in exstrophy bladder patients is reported to be around $15 \%$ after primary bladder closure. In neglected cases, the stones may become very large and infected. Primary bladder calculi result from the stasis of urine but secondary calculi originate from crystallisation of urinary components around a foreign body.

\section{Informed Consent}

It was given by the patient's parents.

\section{FINAL DIAGNOSIS}

Followup case of stage 1 exstrophy closure with B/L multiple ureteric calculi with giant bladder stone with epispadias.

\section{Conclusion}

In conclusion, this case is unusual in that a patient presented with UB calculi and bilateral ureteric calculi that manifested as a palpable mass associated with symptoms of urinary obstruction and infection. Formation of bladder calculi has been described after successful repair of bladder exstrophy; however, UB calculi along with bilateral ureteric calculi in operated case of bladder exstrophy has been very rare. Treatment options are based on stone size, shape, location, and associated anatomical pathology. After surgical removal and correction, our patient had a favourable outcome on followup with correction of voiding symptoms and without recurrence of calculi to date.

Urinary calculi formation in exstrophy bladder patients is reported to be around 15\% after primary bladder closure. In neglected cases, the stones may present with large urinary bladder calculus and bilateral multiple ureteric calculi. The importance of the report is that it highlights a rare and late complication of primary exstrophy closure.

\section{REFERENCES}

[1] Lattimer JK, Smith MJK. Exstrophy closure: a follow on 70 cases. J Urol 1966:95(3):356-9.

[2] Lancaster PAL. Epidemiology of bladder exstrophy: a communication from the International Clearinghouse for birth defects monitoring systems. Teratology 1987;36(2): 221-7.

[3] Ives E, Coffey R, Carter CO. A family study of bladder exstrophy. J Med Genet 1980:17(2):139-41. 